\title{
Electrogelation and rapid prototyping of Bombyx mori silk fibroin
}

\author{
Shu-Dong Wang ${ }^{\text {a }} \mathrm{b}^{*}$, Ke-Qin Zhang ${ }^{\text {a }}$
}

${ }^{a}$ National Engineering Laboratory for Modern Silk, College of Textile and Clothing, Soochow University, Suzhou, Jiangsu 215123, PR China

${ }^{b}$ College of Textile and Clothing, Yancheng Institute of Industry Technology, Yancheng, Jiangsu 224005, PR China

Abstract Silk fibroin (SF) hydrogel is obtained from the SF aqueous solution, ethanol treatment and ultraviolet photocuring are used for rapid prototyping of the SF electrogels. Shear rheology, morphology, mechanical properties and mechanism of the rapid prototyping of SF electrogels are investigated by rheometer, scanning electron microscopy (SEM), fourier transform infrared spectrometer (FTIR), X-ray diffraction (XRD), and tensile strength tester. The results show that ethanol treatment and ultraviolet photocuring are two appropriate methods for rapid prototyping of the SF electrogels.

Key words biomaterials; porous materials; microstructre

\section{Introduction}

Bombyx mori silk extracted from silkworm is a biopolymer fiber which has been extensively used in textiles for thousands of years [1-3]. Native Bombyx mori silk is composed of SF protein coated with silk sericin proteins. Due to favorable biocompatibility and minimal inflammatory reaction [4], SF has been fabricated into a variety of silk-based materials, such as gels, fibers and films and so on [5-10], and these silk-based materials have been widely used for tissue engineering scaffolds, drug delivery, photonic crystal materials and electronic device [11-14].

SF gels have been gained great interesting due to their high water content, biocompatibility and good diffusive properties with respect to oxygen, nutrients and waste products [15]. SF gels can be produced through a variety of mechanisms, such as vortexing [16], sonication [17], application of direct electrical current [18] and lowering the $\mathrm{pH}$ [19]. Among these methods of gelatin, adhesive gels could be obtained effectively by electrogelation. For electrogelation, electrodes are immersed in the SF aqueous solution and direct current (DC) is applied to the electrodes. SF gels could be collected gradually on the surface of positive electrode. Leisk [20] found that electrogel was reversible if the polarity of the voltage was switched.

As tissue engineering scaffolds, SF gels should have appropriate mechanical properties and structural stability. However, SF gels prepared by the above methods exhibit slow formation and unstable structure. Therefore, it is necessary to improve the structure stability of SF gels through rapid prototyping. In this research, organic alcohol treatment and ultraviolet (UV) photocuring are used for the rapid prototyping of the SF electrogels. Dynamic oscillatory shear rheology, morphology, mechanical properties and mechanism of the rapid prototyping of SF electrogels are investigated.

\section{Materials and Methods}

$\mathrm{SF}$ aqueous solution (SF1) was prepared from the silkworm cocoons according to our previous research [10]. Electrodes (graphite sheet, $10 \times 1 \times 0.2 \mathrm{~cm}$ ) were immersed in the $\mathrm{SF} 1$ and $25 \mathrm{~V}_{\mathrm{DC}}$ was applied over a 4 minute period to the electrodes. SF electrogels (SF2) were gradually collected onto the surface of the positive electrode. The SF2 were immersed in the dehydrated alcohol for 5 minute (with bath ratio of 1:10), and then the ethanol treated SF electrogels (SF3) were kept in distilled water to replace ethanol with water until further experiments. For preparing the UV photocuring SF electrogels (SF4), the SF2 were mixed with $0.1 \mathrm{wt} . \%$ photoinitiator Irgacure (I-2959) and 5 wt.\% poly(vinyl alcohol) (PVA, polymerization 2400) aqueous solution (30 w/v \%), the blended system was mixed thoroughly, then the blended gels were polymerized with UV light $(365 \mathrm{~nm})$ for $1 \mathrm{~min}$ at an intensity of $60 \mathrm{mWcm}^{-2}$. The photocuring scaffold was kept in the boiled water to remove the residual PVA and dried to obtain SF4. The SF1, $\mathrm{SF} 2, \mathrm{SF} 3$ and SF4 were placed at $-20{ }^{\circ} \mathrm{C}$ for about $12 \mathrm{~h}$, and then lyophilized for about $48 \mathrm{~h}$ to achieve freeze-dried samples.

Rheological properties of the SF electrogels were carried out with a RS 6000 rheometer (Thermo Haake) using a

\footnotetext{
* Corresponding author. Email address :sdwang1983@163.com (S.D. Wang), kqzhang@ suda.edu.cn (K.Q. Zhang).
} 
parallel plate (PP20H, 20mm diameter, $0.5 \mathrm{~mm}$ gap) configuration at $25{ }^{\circ} \mathrm{C}$ in oscillatory mode. SEM (S4800, Japan) was used to observe the morphology of the freeze-dried SF electrogels at $3 \mathrm{kV}$. Secondary structures were further analyzed by FTIR (Thermo Scientific, FL, USA) and XRD (X'Pert-Pro MPD, PANalytical, Almelo, Holland) based on the freeze-dried samples. Fourier self-deconvolution (FSD) of the infrared spectra covering the amide I region (1595-1705 $\mathrm{cm}^{-1}$ ) was performed by Opus 5.0 software to identify silk secondary structures [21]. The freeze-dried SF gels were cut into $50 \mathrm{~mm}$ length with 100-200 $\mu \mathrm{m}$ thickness. The samples were tested by using automatic tensile tester (model3365, Instron, Boston, USA) to characterize the mechanical properties [10].

\section{Results and discussion}

Process of electrogelation is shown in Fig. 1 (a), it could be seen that under a low DC electric fields (25V), the SF solution begins to gel on the positive electrode immediately. While electrospinning of SF, is performed at voltage potentials as high as $30 \mathrm{kV}$ [22], indicating that the utilization of low DC voltages to prepare SF gel is novel and efficient. About 15g SF2 (Fig. 1 (c)) is colleted when the electric fields is applied to 240s. Fig. 1 (d) and (e) show that after ethanol treatment and UV photocuring, the SF gels become curing. As shown in Fig. 1 (f) and (g), both viscosity and storage modulus of the three SF gels are much higher than those of SF solution, indicating the excellent adhesive of SF treated with electrogelation. Viscosity and storage modulus of the SF3 and SF4 are higher than those of the SF2, which indicates that the physical or chemical treatment may become candidates for rapid prototyping of SF.

SF1 shows an irregular porous leaflike structure (Fig. 2(a)). After electrogelation, the SF2 also exhibits an irregular network and spongelike morphology and interconnected pores. However, the pore sizes in SF2 (Fig. 2 (b)) are smaller than those of the SF1. When the electrogels are treated by ethanol, the SF3 shows weblike structures with fibril micromorphology, the pore sizes are smaller than those of the SF2. Moreover, the structure becomes compact after ethanol treatment and shows a more stable structure. Fig. 2 (e) shows the morphology of the UV photocuring SF electrogels, it could be seen that SF4 shows a relative more regular porous cellular structure. The results investigate that the UV photocuring preserves the stable three-dimensional structure of SF. Furthermore, it could be seen that the pore sizes of SF4 are similar with SF1 and SF2, and are bigger than those of SF3.

There are 11 component bands contributing to the Amide I band contour in SF [23]. The relative percentage of $\beta$-sheets is calculated from the areas of the $\beta$-sheets bands. Fig. 3 (a) and (b) show that the percentage of $\beta$-sheets of SF2 and SF4 increases when compares to SF1, but the change is not significant ( $p>0.05)$. The results indicates the both the electrogelation and UV photocuring do not significantly change the secondary structure of SF. However, the SF electrogel scaffold is tuned from less $\beta$-sheet form $(17.8 \pm 2.1 \%)$ to $\beta$-sheet dominated forms $(45.1 \pm 0.8 \%)$ after ethanol treatment. The results show that the ethanol treatment changes the secondary structure of SF. To further demonstrate the above results, we study the crystal structure of SF. Compared with SF1, SF2 and SF4, a typical $\beta$-sheet crystalline structure by XRD (Fig. 3 (c)) is observed. The crystalline lattice spacing of 10.1 and $4.5 \AA$ appeared at $2 \theta=8.9^{\circ}$ and $20.6^{\circ}$ respectively [24].

Mechanical properties could reflect structural stability and the effects of rapid prototyping. As shown in Fig. 3 (d), the breaking strength (6.5MPa) of the SF scaffold after ethanol treatment increases by more than one times when compares to the as-prepared SF electrogel (3.2MPa), the young' modulus of SF3 (16.7 MPa) is far higher than that of the as-prepared SF electrogel (5.4 MPa), which indicates that the ethanol treated SF electrogel has a good forming effect. Furthermore, we can found the UV photocuring SF electrogel also has a high breaking strength (7.8MPa), the young's modulus (16.6MPa) is a litter smaller than that of the ethanol treated electrogel, which also demonstrates that the UV photocuring can also leads a good gel forming effect. The above results indicate that both ethanol treatment and UV photocuring could lead a good rapid prototyping.

From the results of FTIR and mechanical properties, we find that the breaking strength of SF2 is a litter higher than that of SF1, this is due to the $\beta$-sheet content increases (Fig. 4 (a)). However, the electrogelation does not change the microstructure of SF according to the XRD patterns. According to the results of FTIR and XRD, we conclude that the results of high breaking strength and young' modulus of the ethanol treated SF electrogel are due to the high content of $\beta$-sheet, and the secondary structure of the protein changes significantly (Fig. 4 (b)). As 
shown in Fig. 3 (d), the breaking strength and young' modulus of the UV photocuring increase when compares to the untreated SF electrogel, but since the microstructure of the UV photocuring SF scaffold does not change, therefore, we conclude that the mechanism of rapid prototyping for UV photocuring is physical crosslinking.

\section{Conclusion}

SF hydrogel is prepared successfully by electrogelation, and ethanol treatment and UV photocuring are used for the rapid prototyping of the SF electrogels. Results show that ethanol treatment and UV photocuring are two appropriate methods for rapid prototyping of the SF electrogels. Mechanism of the rapid prototyping indicates that the ethanol treatment changes the secondary structure of the SF. However, the mechanism of UV photocuring for the rapid prototyping is physical crosslinking.

\section{Acknowledgment}

This research was financially supported by the Natural Science Foundation of Jiangsu Province, China (No.BK20131222). The authors are also grateful for the special project of nanotechnology of Suzhou, China (No. ZXG2013037) and agricultural support rogram of Yancheng, China (No.2015028).

\section{Reference}

[1] Shao ZZ, Vollrath F. Nature 2002; 418:741-741.

[2] Ude AU, Eshkoor RA, Zulkifili R, Ariffin AK, Dzuraidah AW, Azhari CH. Mater Design 2014; 57:298-305.

[3] Wang SD, Zhang YZ. Fiber Polym 2014; 15:1129-1136.

[4] Wang SD, Zhang YZ, Wang HW, Dong ZH. Int J Biol Macromol 2011; 48:345-353.

[5] Ma YF, Feng QL, Bourrat X. Mater Sci Eng C 2013; 33:2413-2420.

[6] Ming JF, Bie SY, Jiang ZJ, Wang P, Zuo BQ. Mater Lett 2014; 126:169-173.

[7] Kundu B, Rajkhowa R, Kundu SC, Wang XG. Adv Drug Deliver Rev 2013; 65:457-470.

[8] Xiao SL, Wang ZJ, Ma H. Adv Powder Technol 2014; 25:574-581.

[9] Um IC, Kweon H, Park YH. Int J Biol Macromol 2001; 29:91-97.

[10] Wang SD, Zhang YZ, Wang HW, Yin GB, Dong ZH. Biomacromolecules 2009; 10:2240-2244.

[11] Yin GB, Zhang YZ, Wang SD, Shi DB, Dong ZH, Fu WG. J Biomed Mater Res A 2010; 93:158-163.

[12] Kundu B, Saha P, Datta K, Kundu SC. Biomaterials 2013; 34:9462-9474.

[13] Lawrence BD, Cronin-Golomb M, Georgakoudi I, Kaplan DL, Omenetto FG. Biomacromolecules 2008; 9:1214-1220.

[14]Yun YS, Cho SY, Shim J, Kim BH, Chang SJ, Baek SJ, Huh YS, Tak Y, Park YW, Park S, Jin HJ. Adv Mater 2013; 25:1993-1998.

[15] Xiao W, He J, W. Nichol J, Wang L, B. Hutson C, Wang B, Du Y, Fan H, Khademhosseini A. Acta Biomater 2011; 7:2384-2393.

[16] Rockwood DN, Pread RC, Yucel T, Wang XQ, Lovett M, Kaplan DL. Nat Protoc 2011; 6:1612-1631.

[17] Wang XQ, Kluge JA, Leisk GG, Kaplan DL. Biomaterials 2008; 29:1054-1064.

[18] Lu Q, Huang YL, Li MZ, Zuo BQ, Lu SZ, Wang JN, Zhu HS, Kaplan DL. Acta Biomater 2011; 7:2394-2400.

[19] Fang J, Zhang X, Cai Y, Wei Y. Biomacromolecules $2011 ; 12$ :1578-1584.

[20] Leisk GG, Lo TJ, Yucel T, Lu Qiang, Kaplan DL. Adv Mater 2010; 22:711-715.

[21] Bai SM, Zhang WM, Lu Qiang, Ma QH, Kaplan DL, Zhu HS. J Mater Chem B 2014; 2:6590-6600.

[22] Wang SD, Zhang YZ, Yin GB, Wang HW, Dong ZH. Mater Sci Eng C 2010; 30:670-676.

[23] Hu X, Kaplan D, Cebe P. Macromolecules 2006; 39:6161-6170.

[24] Zhang Q, Li M, Xu W, Li J, Yan S. Mater Lett 2013; 105:8-11. 

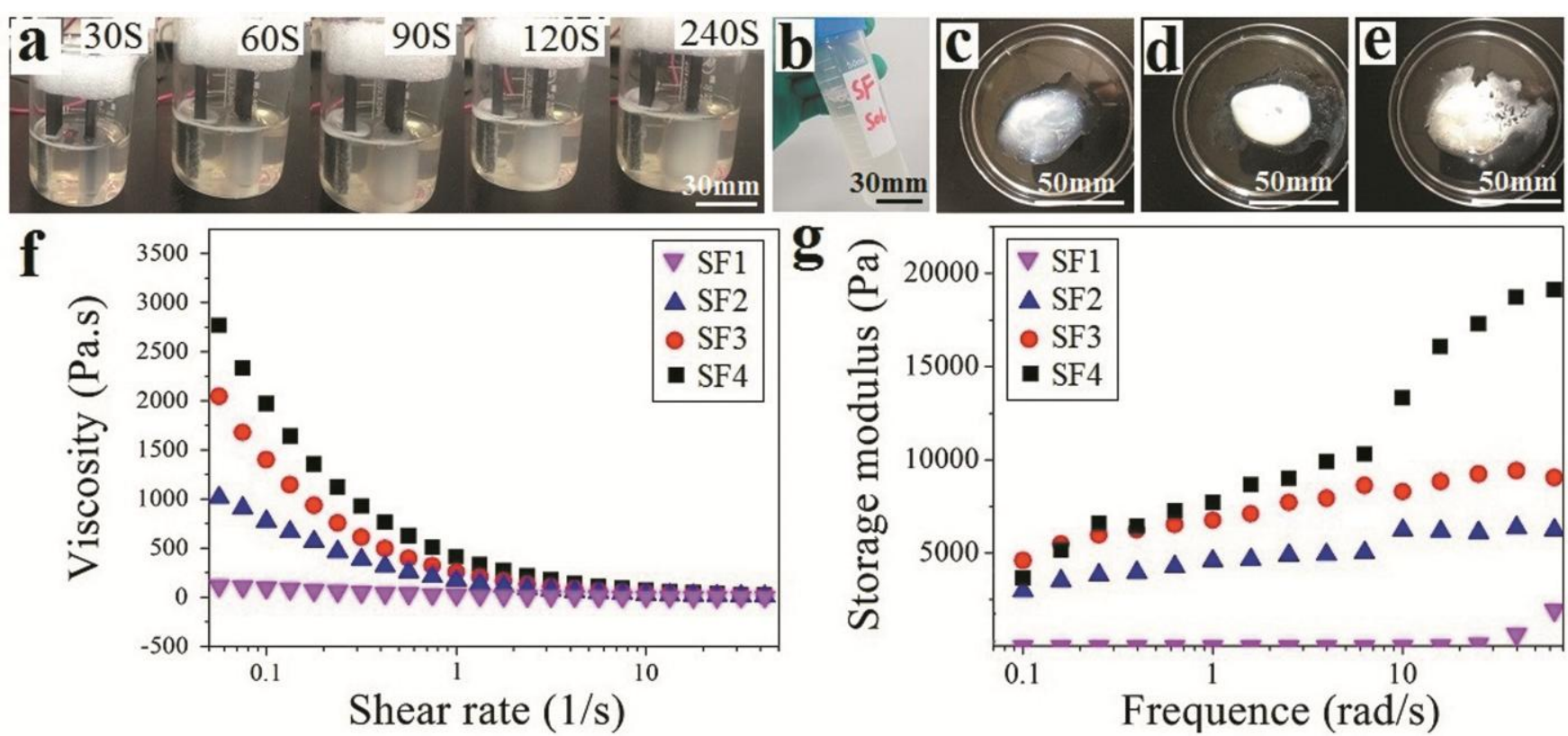

Fig. 1. (a) Electrogelatin of the SF solution, photomacrographs of SF1 (b), SF2 (c), SF3 (d), and SF4 (e), viscosity (f) and storage modulus $(\mathrm{g})$ of the four kinds of SF. 

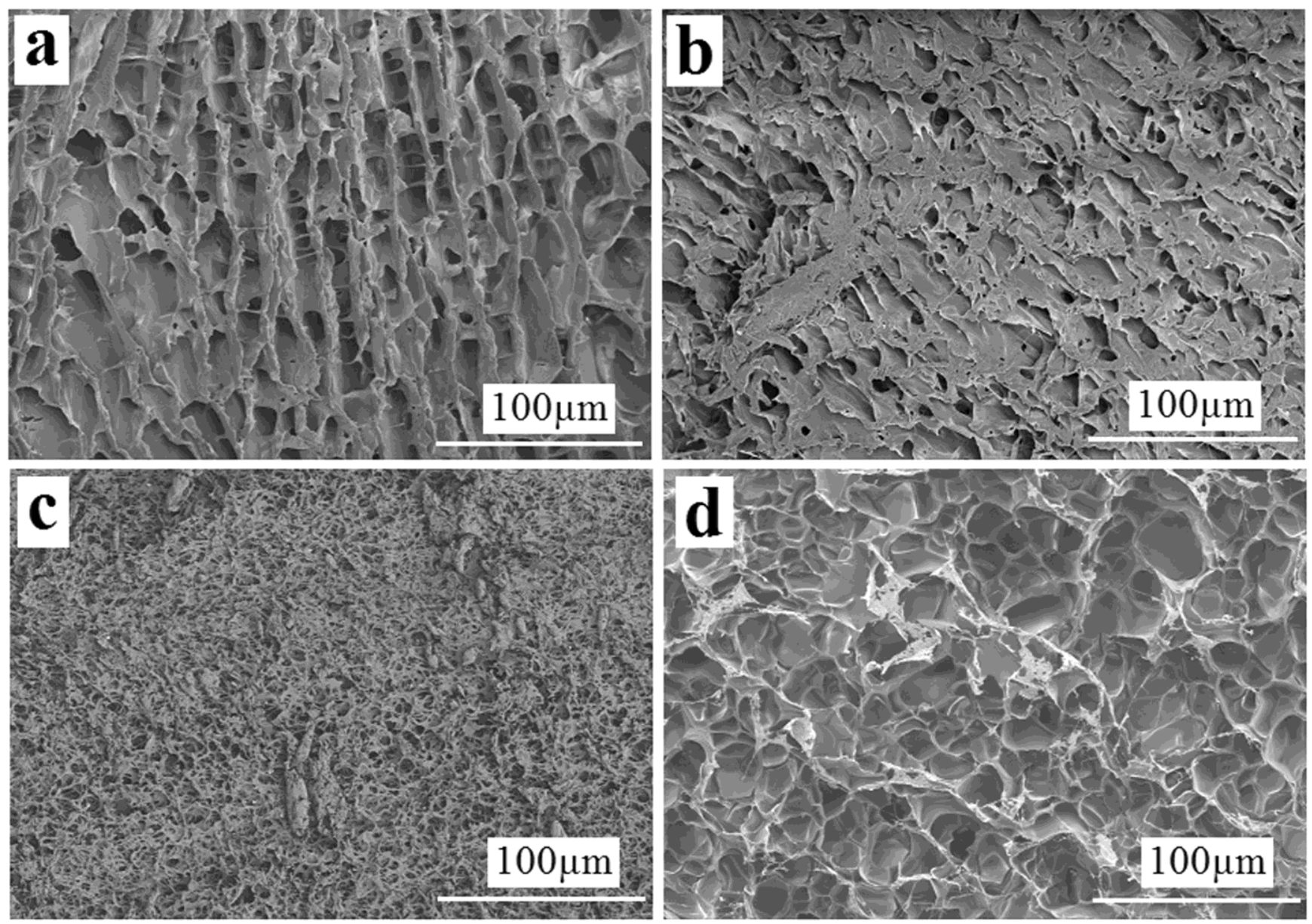

Fig. 2. SEM images of the SF1 (a), SF2 (b), SF3 (c), and SF4 (d). 

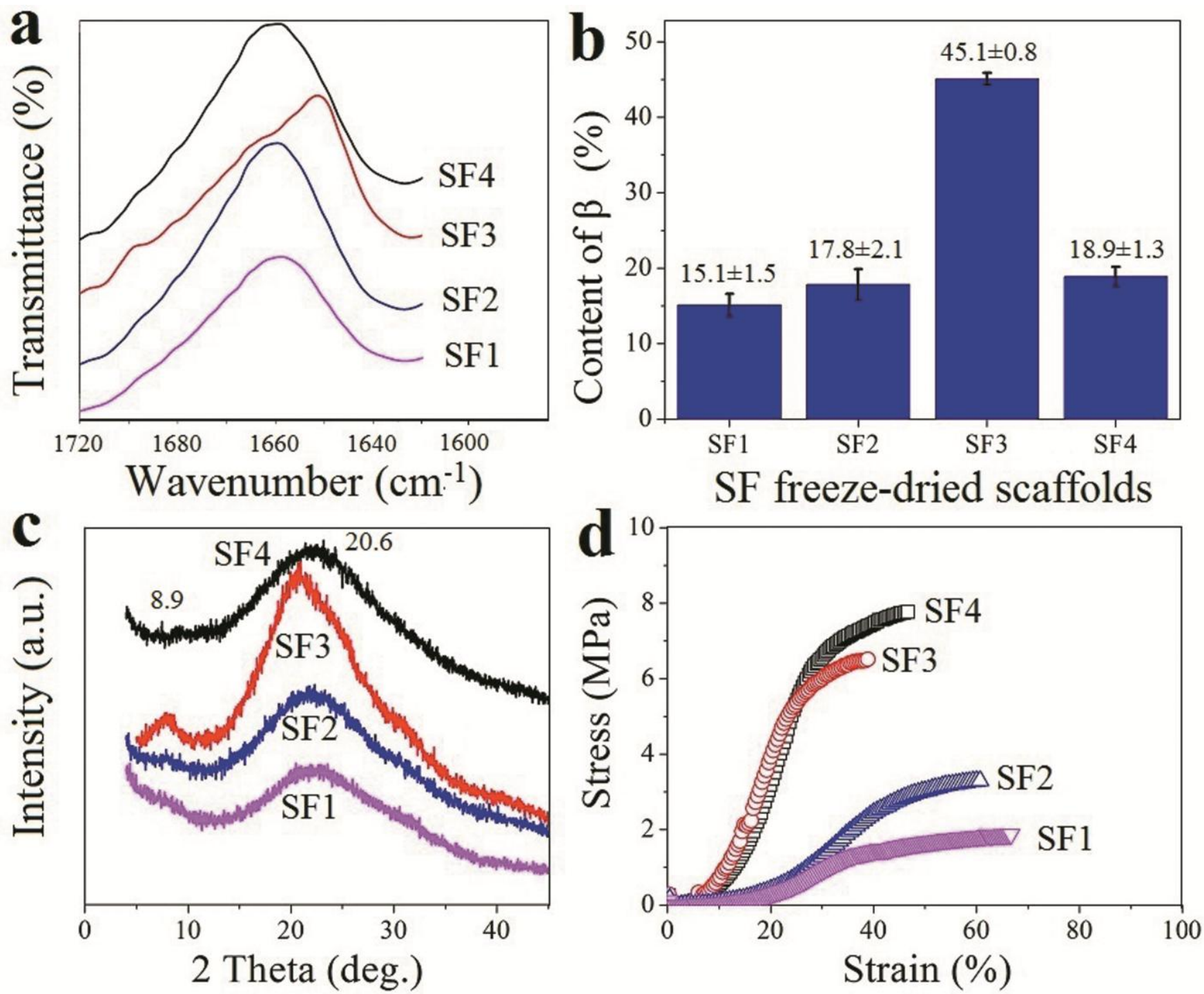

Fig. 3. (a) FTIR spectra, (b) percentage of $\beta$-sheet content, (c) XRD patterns, and (d) stress-strain curves of the SF scaffolds. 

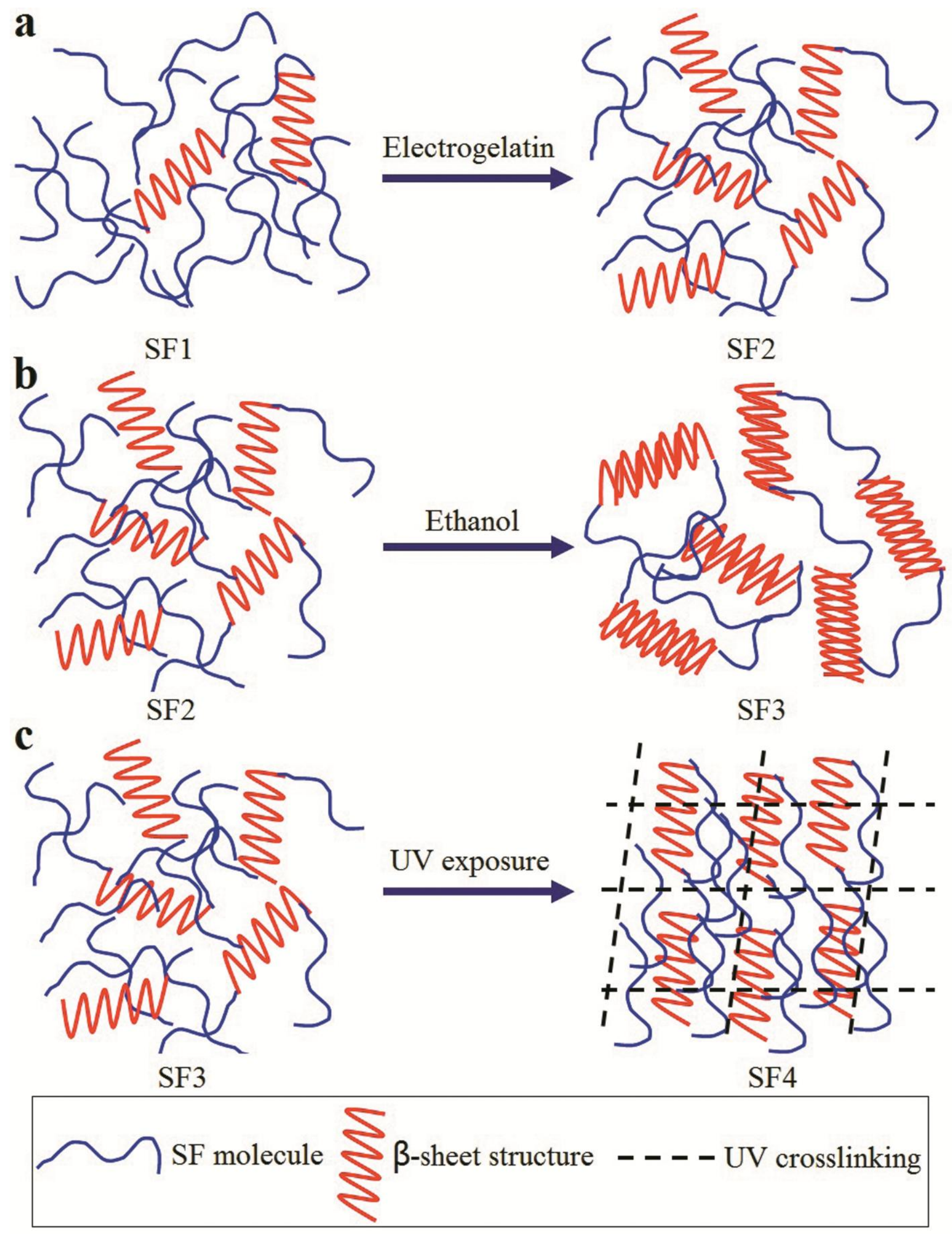

Fig. 4. Schematic for mechanism of the electrogelatin and rapid prototyping of SF. 